\title{
Nasopalatine duct cyst with sebaceous differentiation: a rare case report with literature review
}

\author{
Han-Gyeol Yeom ${ }^{1}$ (D) Jae-Hyun Kang ${ }^{2} \mathbb{D}$, Sun-Ung Yun ${ }^{3}$ (D) and Jung-Hoon Yoon ${ }^{*}$ (D)
}

\begin{abstract}
Background: The aim of this study was to report a rare case of nasopalatine duct cyst with sebaceous differentiation. Further, a systematic search of the literature was performed to identify studies reporting patients with intraosseous jaw cysts with sebaceous differentiation.

Case presentation: A 55-year-old Korean man was referred to our hospital because of a cystic lesion of the anterior maxilla. Radiologic examination revealed a well-circumscribed radiolucent lesion in the anterior maxilla. Histology showed a respiratory columnar and cuboidal epithelium-lined cyst. Transition from the ciliated columnar epithelium to stratified squamous epithelium with sebaceous differentiation was observed. Based on these findings, the final diagnosis was nasopalatine duct cyst with sebaceous differentiation. A systematic search of the literature was performed to identify studies reporting patients with intraosseous jaw cysts with sebaceous differentiation. There were 24 cases of sebaceous differentiation in the epithelium of the cysts including 2 odontogenic keratocysts, 8 orthokeratinized odontogenic cysts, 8 dentigerous cysts, 1 radicular cyst, and 2 glandular odontogenic cysts. However, no case reports describing the occurrence of nasopalatine duct cysts with sebaceous differentiation have been reported.
\end{abstract}

Conclusion: This first case report of nasopalatine duct cysts with sebaceous differentiation could provide insight into the diagnostic process of cystic lesions with sebaceous differentiation.

Keywords: Nasopalatine duct cyst, Incisive canal cyst, Sebaceous differentiation

\section{Background}

Nasopalatine duct cysts (NPDCs), also known as incisive canal cysts, are the most common non-odontogenic developmental cysts in the jaws $[1,2]$. As the lesions are usually asymptomatic, NPDC is discovered mostly on routine panoramic radiographs [2]. Histologically, squamous, ciliated (respiratory), and cuboidal epithelium are found in these cysts. More than one epithelial type is commonly observed, and the type of epithelium depends

\footnotetext{
*Correspondence: opathyoon@wku.ac.kr

${ }^{4}$ Department of Oral and Maxillofacial Pathology, Daejeon Dental

Hospital, Wonkwang University College of Dentistry, Daejeon, Korea

Full list of author information is available at the end of the article
}

on the location involved (palatine, nasal, or intermediate) $[1,2]$. The etiology and pathogenesis of these cysts are unknown, but some investigators have proposed that NPDCs develop from the spontaneous proliferation of the remnants of embryonic tissue [1-4]. Epithelial remnants of the nasopalatine duct may be stimulated to proliferate by trauma, infection, or mucous retention $[1,2$, 4]. As the cysts have been found in human fetal incisive canals, spontaneous cystic degeneration of epithelial remnants is also suggested $[1,2]$.

Sebaceous glands are prominent skin appendage components formed in close association with or independent of hair follicles $[5,6]$. Sebaceous glands are generally found in most parts of the body [6] and are 
very common in the oral mucosa $[5,6]$. Within the oral cavity, sebaceous glands may present as small, yellowish spots called Fordyce granules, which exhibit a predilection for the buccal mucosa [7-9].

Some cases of intraosseous jaw cysts with sebaceous differentiation have been reported [10-16]. There were 24 cases of sebaceous differentiation in the epithelium of the cysts including odontogenic keratocysts (OKCs), orthokeratinized odontogenic cysts (OOCs), dentigerous cysts (DCs), radicular cysts (RCs), and glandular odontogenic cysts (GOCs). However, we found no case report describing the occurrence of NPDC with sebaceous differentiation in our search of the English literature. Here, we report a rare case of NPDC with sebaceous differentiation. In addition, a systematic search of the literature was performed to identify studies reporting patients with intraosseous jaw cysts with sebaceous differentiation.

\section{Case presentation}

A 55-year-old Korean man was referred to our hospital from a local dental clinic because of a cystic lesion in the anterior maxilla. The patient had no pain or significant systemic disease.

Clinical examination revealed no distinct expansion of the anterior maxilla. Left maxillary incisors were lost. Panoramic radiography revealed a well-circumscribed radiolucent lesion in the anterior maxilla (Fig. 1a). The border of the lesion was well-defined, with a corticated margin. No apparent external root resorption of the adjacent teeth was observed, and no normal nasopalatine canal structures were observed.

Additional cone-beam computed tomography scans revealed a well-defined corticated lesion. As the lesion was not large enough to affect the labial or palatal cortical bone, no apparent expansion pattern of the labial and palatal cortex was observed (Fig. 1b, c).

An ovoid-shaped, low-attenuated lesion was observed in the anterior part of the maxilla (yellow arrow). Neither
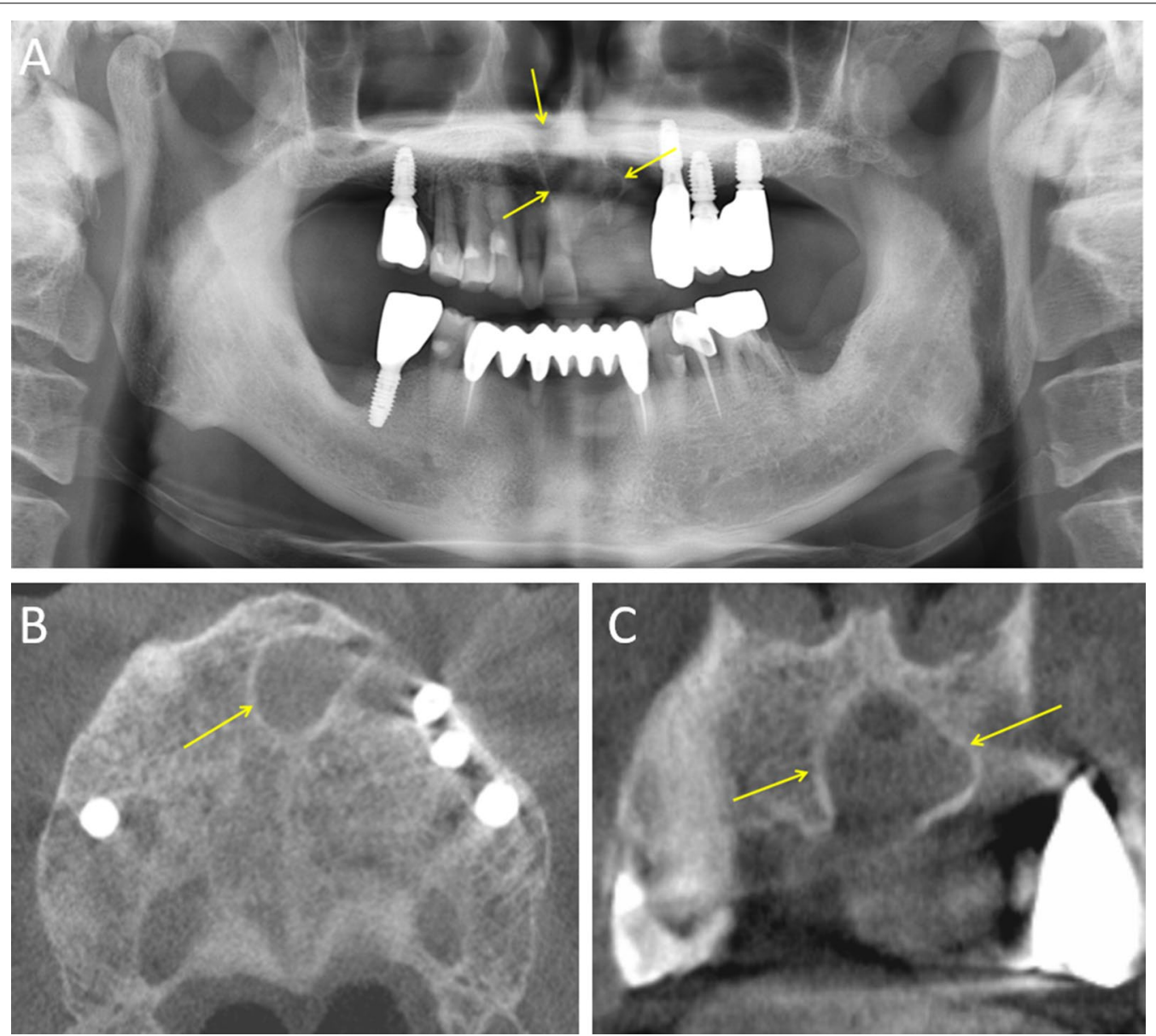

Fig. 1 a Panoramic radiograph showing a radiolucent lesion in the anterior maxilla (yellow arrow). b, c Cone-beam computed tomography scan showing a well-defined corticated cystic lesion. (B. axial, C. coronal) 
an apparent expansion pattern nor deviation of adjacent structures was observed. Radiological and clinical diagnosis of NPDC was made.

Surgical cyst enucleation and histopathological examination were performed. Histopathologically, the lesion consisted of a cuboidal and respiratory ciliated columnar epithelium-lined cyst (Fig. 2a,b). Transition from the ciliated columnar epithelium to stratified squamous epithelium with sebaceous differentiation was observed (Fig. 3). Considering both histopathological and radiographic properties, the final diagnosis made was NPDC with sebaceous differentiation.

\section{Discussion and conclusion}

A systematic search of the literature was performed to identify studies reporting patients with intraosseous jaw cysts with sebaceous differentiation. The PubMed/MEDLINE/Google Scholar databases and gray literature were searched for English language papers using a combination of terms such as "intraosseous" or "jaw" or "maxilla (maxillary)" or "mandible (mandibular)," "cyst" or "cystic lesion" and "sebaceous." The literature from 1980 to 2020 was searched. Papers that allowed access only to the abstract were excluded.

As a result, a total of 88 papers were reviewed, and finally, seven papers, including case series, case reports, and articles investigating the imaging and histopathologic appearance of cystic lesions [10-16], with 24 patients were selected. Table 1 summarizes the data obtained from the selected papers.

There were 24 cases of sebaceous differentiation in the epithelium of the cysts, but no case of sebaceous differentiation in NPDC was reported. Out of the 24 cases, there were $8(33 \%)$ cases of OOC and DC, respectively, $5(21 \%)$ cases of OKC, $2(8 \%)$ cases of GOC, and $1(4 \%)$ case of RC. Sebaceous elements in the cystic epithelium probably represent sebaceous metaplasia [11]. Various

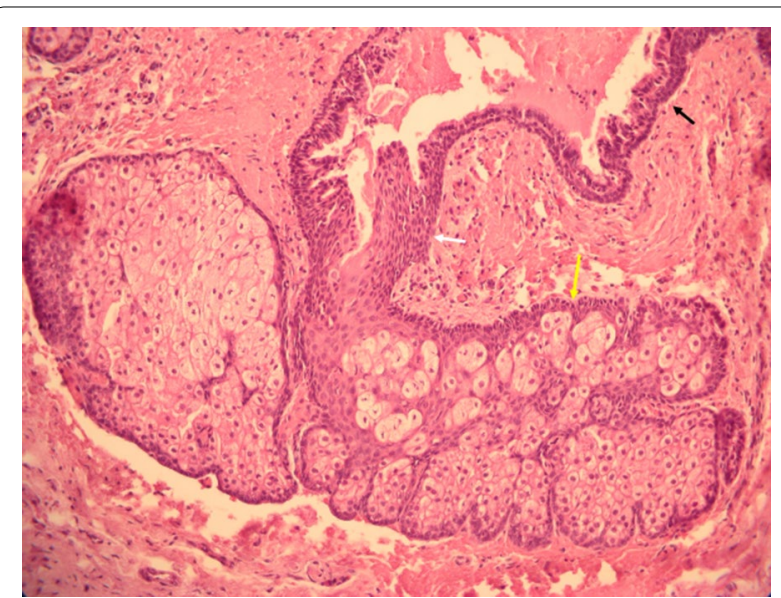

Fig. 3 The lining epithelium showed a transition from the ciliated columnar epithelium (black arrow) to stratified squamous epithelium (white arrow) with sebaceous differentiation (yellow arrow)

hypotheses have been suggested for the etiopathogenesis. One hypothesis is that the origin might be the sequestered multipotent epithelial cells that aid in the development of the oral cavity, which may have been embedded deep in the surface and entrapped in the developing jaw during embryogenesis. Hence, its proliferation might have been induced by dental inflammation, trauma, or cystic change $[15,17,18]$. Another hypothesis is that cysts develop from the existing or cystic epithelium that undergoes dermal metaplasia $[15,17,18]$. Such theories can also be used to understand the sebaceous differentiation of NPDCs.

We proposed three possibilities for the etiology of this case. First, because of the chronic periodontitis or unknown traumas, sebaceous differentiation of entrapped multipotent epithelial cells occurred, and this was simply adjacent to the NPDC. Second, the NPDC
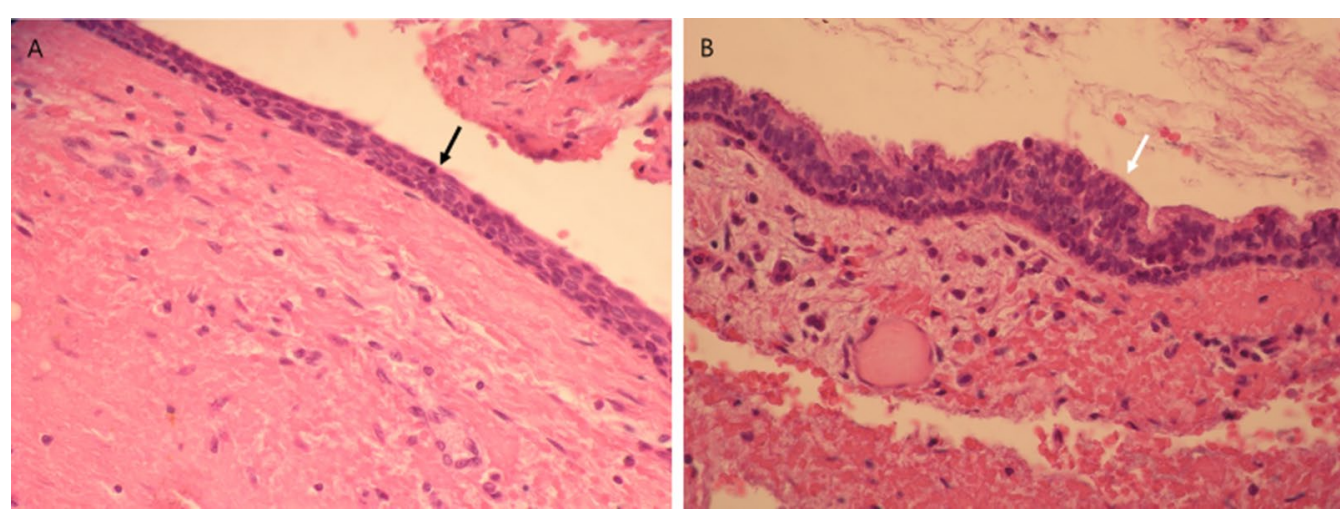

Fig. 2 a, b The cyst is lined by cuboidal epithelium (A, black arrow) and ciliated columnar epithelium (B, white arrow). 


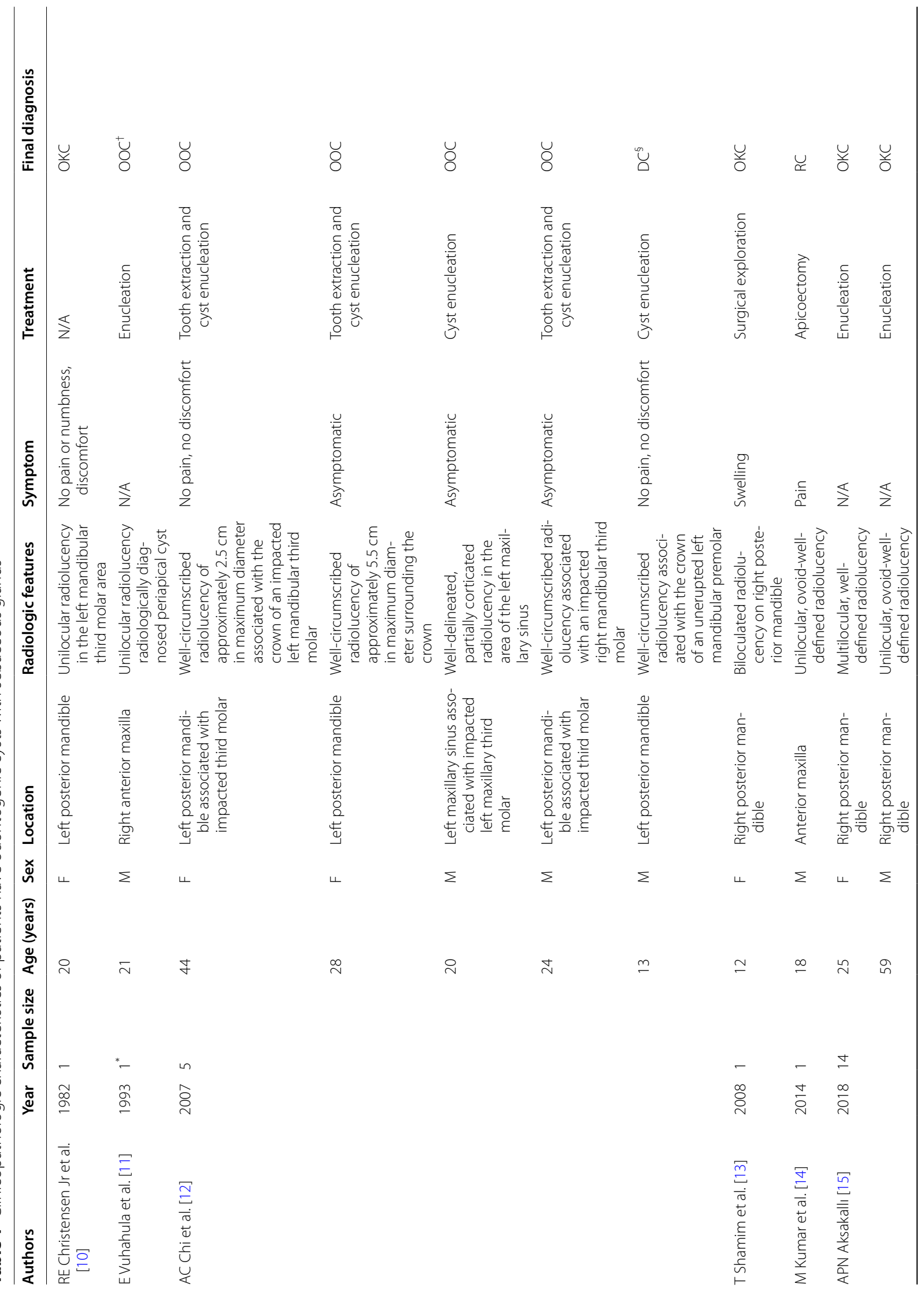




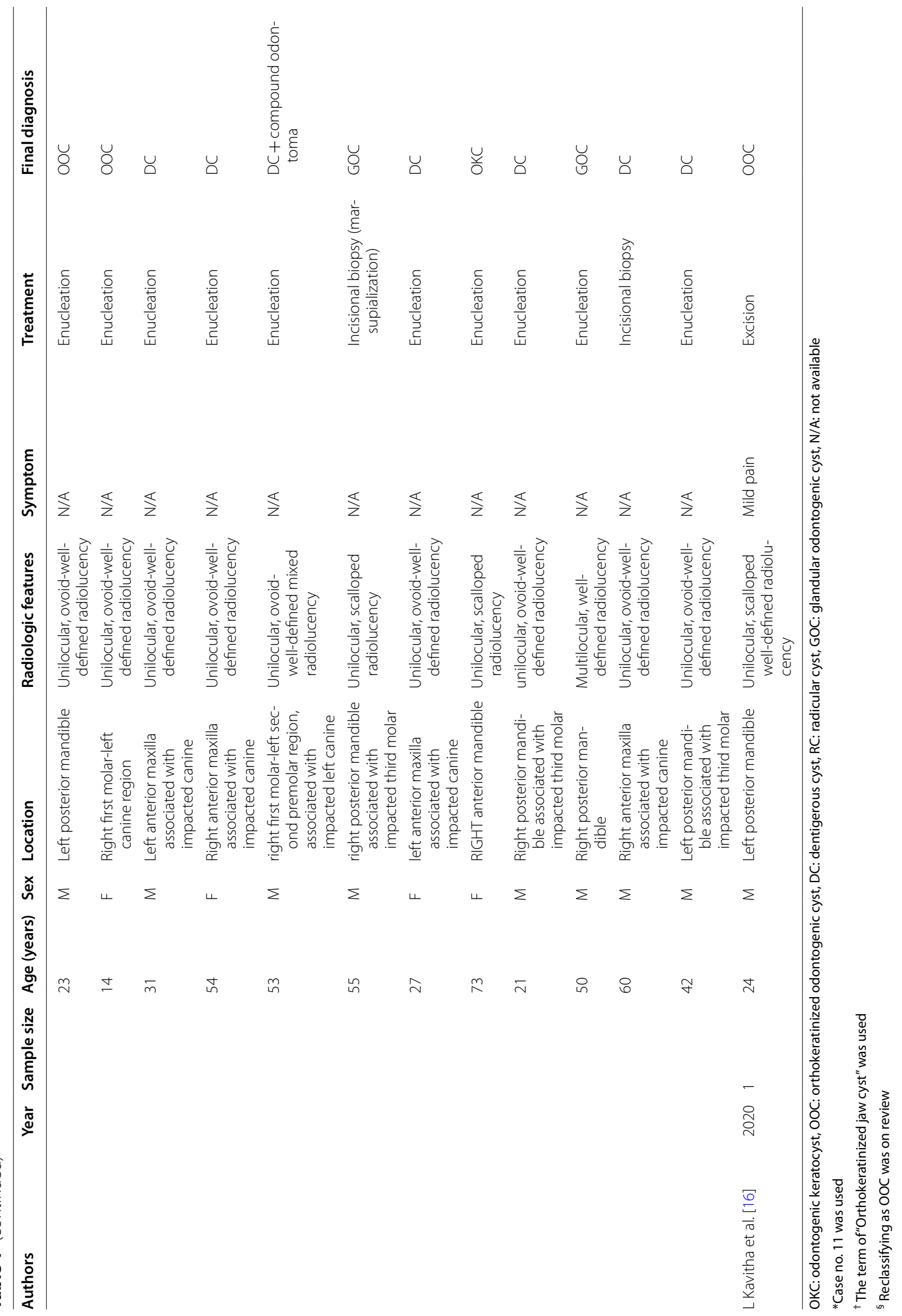


occurred first, and subsequent adjacent multipotent epithelial cells were affected by stimuli of hydrostatic pressure due to the cystic change and underwent sebaceous differentiation. Third, NPDC occurred first followed by sebaceous metaplasia of the cyst lining epithelium.

All three hypotheses are possible, but considering the histopathologic features, the third hypothesis is thought to be the most likely. The sebaceous component was not simply adjacent to the NPDC but was connected along the cyst lining. In addition, a transition from the ciliated columnar epithelium to stratified squamous epithelium was observed in the epithelium where the sebaceous component was connected, so it is most likely that the sebaceous component also occurred among metaplasia in various directions.

In this paper, we described the first case of NPDC with sebaceous differentiation and suggest a possible etiology based on the results of a literature review conducted on the reports of sebaceous differentiation in various intraosseous jaw cysts. Although there are various limitations to the generalization of this study due to the small number of cases, we expect to improve the understanding and diagnosis of intraosseous jaw cysts with sebaceous differentiation by reporting this paper.

\section{Abbreviations}

NPDC: Nasopalatine duct cyst; OKC: Odontogenic keratocyst; OOC: Orthokeratinized odontogenic cysts; DC: Dentigerous cyst; RC: Radicular cysts; GOC: Glandular odontogenic cyst.

\section{Acknowledgements}

Not applicable

\section{Authors' contributions}

This study was conceived by J.H.Y. who also analyzed and interpreted the histopathologic data. H.G.Y. and S.U.Y. interpreted the radiologic data. H.G.Y and J.H.K. wrote the manuscript. All authors read and approved the manuscript.

\section{Funding}

This work was supported by grants from the Foundation of Wonkwang University in 2021. The funder had no role in the design of the study, the collection, analysis and interpretation of the data, and in writing the manuscript.

\section{Availability of data and materials}

All data analyzed during this study are included in this published article.

\section{Declarations}

\section{Ethics approval and consent to participate}

The study was approved by the Institutional Review Board of Daejeon Dental Hospital, Wonkwang University (IRB no. W2101/003-001). Written informed consent was obtained from the patient for participation.

\section{Consent for publication}

Written informed consent was obtained from the patient for publication of this case report and any accompanying images.

\section{Competing interests}

The authors declare that they have no competing interests.

\section{Author details}

'Department of Oral and Maxillofacial Radiology, Daejeon Dental Hospital, Wonkwang University College of Dentistry, Daejeon, Korea. ${ }^{2}$ Department of Oral and Maxillofacial Surgery, Daejeon Dental Hospital, Wonkwang University College of Dentistry, Daejeon, Korea. ${ }^{3}$ Department of Oral and Maxillofacial Surgery, Yuseong Sun Dental Hospital, Daejeon, Korea. ${ }^{4}$ Department of Oral and Maxillofacial Pathology, Daejeon Dental Hospital, Wonkwang University College of Dentistry, Daejeon, Korea.

Received: 26 April 2021 Accepted: 5 August 2021

Published online: 26 August 2021

\section{References}

1. Swanson KS, Kaugars GE, Gunsolley JC. Nasopalatine duct cyst: an analysis of 334 cases. J Oral Maxillofac Surg. 1991;49(3):268-71.

2. Escoda Francolí J, Almendros Marqués N, Berini Aytés L, et al. Nasopalatine duct cyst: report of 22 cases and review of the literature. Med Oral Patol Oral Cir Bucal. 2008;13(7):E438-43.

3. Lake S, Iwanaga J, Kikuta S, et al. The incisive canal: a comprehensive review. Cureus. 2018;10(7):e3069.

4. Vasconcelos RF, Ferreira de Aguiar MC, Castro WH, et al. Retrospective analysis of 31 cases of nasopalatine duct cyst. Oral Dis. 1999;5(4):325-8.

5. Izutsu T, Kumamoto H, Kimizuka S, et al. Sebaceous adenoma in the retromolar region: report of a case with a review of the English literature. Int J Oral Maxillofac Surg. 2003;32(4):423-6.

6. Thody AJ, Shuster S. Control and function of sebaceous glands. Physiol Rev. 1989;69(2):383-416.

7. Neville BW, Damm DD, Allen CM, et al. Oral and maxillofacial pathology. 4th ed. Philadelphia: Saunders; 2016. p. 6-7.

8. Miles AE. Sebaceous glands in the lip and cheek mucosa of man. Br Dent J. 1958:105:235-48

9. Halperin V, Kolas S, Jefferis KR, et al. The occurrence of Fordyce spots, benign migratory glossitis, median rhomboid glossitis, and fissured tongue in 2478 dental patients. Oral Surg Oral Med Oral Pathol. 1953;6:1072.

10. Christensen Jr, Russell E, Robert HP. Intraosseous mandibular cyst with sebaceous differentiation. Oral Surg Oral Med Oral Pathol 1982; 53(6): 591-595.

11. Vuhahula $\mathrm{E}$, Nikai $\mathrm{H}$, ljuhin $\mathrm{N}$, et al. Jaw cysts with orthokeratinization: analysis of 12 cases. J Oral Pathol Med. 1993;22(1):35-40.

12. Chi AC, Neville BW, McDonald TA, et al. Jaw cysts with sebaceous differentiation: report of five cases and a review of the literature. J Oral Maxillofac Surg. 2007;65(12):2568-74.

13. Shamim T, Varghese VI, Shameena PM, et al. Sebaceous differentiation in odontogenic keratocyst. Indian J Pathol Microbiol. 2008;51:83-4.

14. Kumar M, Modi TG, Bajpai M, et al. Rare presentation of radicular cyst with sebaceous differentiation. Saudi J Oral Sci. 2014;1:120-2.

15. Nihan A. Sebaceous glands within odontogenic cysts Odontojen kistlerde yağ bezi lobulüsü. Margins. 2018;2:14

16. Kavitha L, Kavitha B, Sivapathasundharam B. Fortuitous sebaceous glands in orthokeratinized odontogenic cyst (OOC)-a case report and review of literature. Eur J Mol Clin Med. 2020;7(9):1037-44.

17. Allon DM, Calderon S, Kaplan I. Intraosseous compound-type dermoid cyst of the jaw: case report. IJHNS. 2010;1:103-6.

18. Spouge JD. Sebaceous metaplasia in the oral cavity occurring associated with dentigerous cyst epithelium. Oral Surg Oral Med Oral Pathol Oral Radiol Endod. 1996;21:492.

\section{Publisher's Note}

Springer Nature remains neutral with regard to jurisdictional claims in published maps and institutional affiliations. 